\title{
Quelques résultats de l'expédition antarctique belge
}

In: Annales de Géographie. 1901, t. 10, n54. pp. 454-461.

Citer ce document / Cite this document :

Zimmermann Maurice. Quelques résultats de l'expédition antarctique belge. In: Annales de Géographie. 1901, t. 10, n54. pp. 454-461.

doi : $10.3406 /$ geo.1901.7026

http://www.persee.fr/web/revues/home/prescript/article/geo_0003-4010_1901_num_10_54_7026 


\section{QUELQUES RESLLTATS \\ DE L'EXPEDITION ANTARCTIQLE BELGE}

Nous possridons aujourd'hui assez de renseignements pour être en mesure de juger lólendue des travaux de l'expédition de Gerlache'. Son promoteur en avait concu lidée des 189 ' , et il lui fallut frois ans, au prix de mille difficultes, pour équiper son navire, assurer son personnel et surtout faire face à ba grosse depense de 300000 fro, minimum nécessaire pour couvrir tous les frais. Malgré la générosite de quelques particuliers, les subventions de l'Etat belge, les souscriptions ef les fotes, les fonds furent tout juste suftisants ${ }^{2}$. Rien n'avait été négligé sams doute, il convient de le: dire et de détruire à cet égard des idées qui enrent coms pendant un temps, pour liquipement nautique du navire. La "Belgica "stait un ancien troismâts-barque norvegien, de $33 \mathrm{~m}$. 80 de long, de $7 \mathrm{~m} .80$ de large, jaugeant $336 \mathrm{t}$; ; il avait éte complitement remis à neuf pour le voyage, pourvu d'une. machine de 130 chevaux, et muni d'une série de défenses très ingénieust:ment appropriées contre les glares.

Contrairement à ce qui a ćle soutenu, il n'avait point éte renforcé intrrieurement a la maniere du "Fram " de Nansen, mais on a vait pourvu la coque: entière d'un revêtement continu de "greenheart ", bois tropical à la fois dur et élastique; l'avant avait été arrondi, de facon que le bateau pût se. dresser sur les glaces et les écraser de son poids : toute la membrure prisentait une épaisseur plus considérable gu'il n'est coutume, et l'avant se trouvait protégé par une paroi de $3 \mathrm{~m}$. 60, l'arrice par une paroi de $1 \mathrm{~m}$. 30 . Des bandes de fonte étaient destinées en outre a subir le memier choc des blocs anguleux et à en émousser les aspérités.

C'étaient lì des précautions capitales, comme la suite du voyage I" proura. Il est peu probable qu'un bateau moins solide se fût tiré du péril quecourut la "Belgica" au début de sa course, lorsqu'elle donna sur une roche du canal du Beagle, ni surfout qu'il ê̂t résisti à la violente pression des glaces du 31 mai 1899. Elle fut soumise, en effet, à l'epreuve redoulable d'un détention de 12 mois $1 / 2$ (28 février 1898-14 mar's 1899) dans le park antarctique, beaucoup plus mobile que le pack arctique. Sans doute le champ de glace, où elle se trouvat enserrée témoigna d'une résistance particulière, et assura à l'expédition un hivernage tranquille, mais dans certaines circonstances le navire dut déployer des qualités de force tris grandes, notamment dans la lutte supreme pour s'affianchir des glaces, lorsquil se troura exposé, sur la lisiere du park, aux terribles chocs des glacons soulevés par. la houle.

La "Belgica" poursuivait un but purement scientifique, ce qui ne s'était. pas vu depuis le dernier voyage de James Clark Ross en 1843. Mr de Ger-

1. Voir la Chionique des Annales, VI, 15 novembre 189\%, p. 476, ot surtout VIII, 15 juillet 1899 , j. 383-384.

2. Ce qui laissa le plus a désirer, au dire du Lr Cook, et quiconque lit sa relation sien convaincra aisément, c'est l'équipement proprement polairo de l'expédition. Si elle ết été obligée a un plus long sejour, on s'il avait été necessaire de fairc retraite sur la glace, elle se serait trouve insuffisamment equipece. 
Jache siéait assuré un état-major véritablement de choix, qui, par ses très nombreuses et fort intéressantes publications ${ }^{1}$, a prouvé depuis son retour (novembre 1899) i la fois sa valeur personnelle et le vif intérèt de ses découvertes. L'wurre des déterminations hydrographiques, astronomiques et magnétiques fut poursuivie dans un esprit rigoureusement seientifique par le lieutenant Danco, puis par le second du navire, G. Lecointe. M.Mrs H. Arctowski et E. G. Racovitza se sont révélés de leur côté naturalistes et géographes consommés. Mr Arçtowski particulirrement est en passe de prendre rang parmi les autorités en matière d'océanographie, de météorologie et de glaciologie: polaires.

On duit rependant faire encore une place à part au $D^{\mathbf{r}}$ américain Frederick A. Corok, ancien compagnon de Peary au Groenland, qui le premier de l'expredition, en a publié une relation in extenso sous le titre de Through the First Anturctic Night 1898-1899. A narrative of the voyage of the "Belgica " among newly discovered lands and over an unknoun sea about the South Pole (Lonclon, W. Heinemann, 1900. In-8, xxivt+478 p., 133 pl. de pholog. dont 4 en couleurs. Index. 20 sh.). Cet ouvrage est une des plus remarquables relations polaires qu'il nous ait été donné de lire. Écrivain de race, primesautier el spiritucl, joignant un rare talent descriptif ì une faculté d'observation très aigué, le $\mathrm{D}^{\mathrm{r}}$ Cook nous paraît avoir décrit la vie d'une expédition polaire avec une fidélité, et, si l'on peut dire, une virginité d'impressions bien rares. Il suffit d'ailleurs de jeter un coup d'œil sur ses photographies, extrêmement nombreuses, exécutées el choisies avec art, pour se rendre compte du degré auquel s'élève chez lui le sens du détail raractéristique qui donne la sensation de la rialití. On peut dire que toutes les particularités du domaine glacé où se déroulent les phases de l'expédition se reflètent avec une ctonnante intensité dans cette illustration si originale et si habilement composée. Il suffit de feuilleter les pages pour avoir une vision du voyage. Nul n'avait jamais pensé à photographier des létails aussi spéciaux et cependant aussi typiques que l'aspect d'un trou de phoque ou de cétacé, lia trace d'un manchot sur la neige, des intervieus de manchots, certaines formes etrangement usées d'icebergs (en forme de baleine, en forme de meule). Beaucoup de ces photographies ont une incomparable portéc géographique : telles l'aspect d'une jeune et d'une viejlle rrevasse de la banquise, les hummocks et les cestrugi ${ }^{2}$, la vue i vol cloiseau de la banquise

I. Lire la bibliographie de ces publications id la fin de l'article de Mr GMLE Racovitza, Risultats giniraux de l'expidition antarctique belfe (La Géorraphie, 1, 1900, p. 81-92, carte à 1 : 620000 pl. Iv j. Depuis lors a paru dans"lo Bulletin de la Sociétr royale Belge de Grographie (24" annéc, $1900, \mathrm{n}^{\circ} 1$, p. 7-230), un véritable volume reproduisant quatre conférences de. MMrs G. LFcorste, Apercu des travaus scientifques de I Exprelition antarctique belge (p. 29-52) et l'Hydrographie dans lo ditroit de la Belgica et les obstervations astronomiquex et magnetiques dans la zone: australe,(p. 53-92); II. Arçтowski, Géographie physique de la rigion antarctique visite par l'expédition de la Belgicu (p. 93-175।; H. G. RacovitzA, La vie des animaux et des plantes dans l'Antarctiqu: ( 1 . $177-230$, l fig.). Ces conferences sont accompagnees de 48 planches, graphiques ou photographies, et de 3 cartes de Lecointe, montrant la topographie du détroit de la Belgica, l'itinéraire du navire le long de la banquise et le tracé de sa derive dans la banquise. Mr A. DE Gerlache a de son côté publié uno. Vrite sur les E.cpélition.s qui ont pricédí crlle de la Belgica aux Regions circumpolaires voisines du méridien du cap Horn (5 cartes dans le texte et bibliographie finale;, suivic dune Relation sommaire du voyage de la Belgica (Bull. Soc. R. Belge Géog., $24^{e}$ annce, $1900, n^{\circ} 5$, p. 365-531).

2. On appello cestrugi les trainises do neige accumulées par lo vent contre des hummocks, et rappelant par leur aspect et leur mode de formation les dunes du désert. 
près de son rebord extírieur, les disques rristallins qui donnent naissance à la glace, nombre de vues d'iccbergs, de caps el d'îles glacées, etc. $M^{*}$ Cook a également fait une place importante à la reproduction des rares animaux de ces régions: phoques, manchots, pétrels, goëlands, il a poussé le scrupule jusqu'i photographier de liès près les rares pentes couvertes de lichen et de mousse, les étoiles de mer et les oursins qui peuplent le fond de l'Occian Antarctique, et cette crevette du genre Euphausia, découverte par Racovitza, qui représente la principale nourriture des manchots et des phoques. La vie meme à bord, les particularilés d'aspect de la "Belgica " ot de ses habitants ont été recucillies scrupuleusement. Rien n'est plus curieux à cet égard que la mise en regard des photographies de l'auleur, de $\mathrm{MM}^{\mathrm{rs}}$ Amundsen et Racovilza avant leur départ ef a leur retour. La comparaison en dit plus que n’importe quelle parole sur la rudesse des conditions qu'impose la nature polaire. Le texte, par sa raleur descriptive, lient les promesses des photographies. Entin l'ourrage est complété par soixante pages d'appendices scientifiques (p. 409-468) dus à MMrs Racovitza, Aretowski, Lecointe, Amundsen et Cook, en sorte qu'en attendant les mémoires scientifiques détaillés de la Commission de la "Belgica", celte relation peut servir de manuel. Il ne lui manque que des cartes a grande échelle.

Découvertes topographiques. - Les parages où ont porté les travaux topographiques de l'expédition belge ont été sans aucun doute les plus fréquemment visités de tout le monde antarctique. Cook, lors de son voyage de 1774-7\%, avait signalé la multitude de phoques à fourrures qui hantaient les côtes de la Géorgie du Sud : c'était ourrir la voie aux recherches intéressées des chasseurs. Lorsque, vers le début du siècle, les réserves d'animaux de la Géorgie du Sud furent détruites, l'Anglais William Smyth, par la découverte des Shetlands du Sud (févr. 1819), inaugura une période de voyages annuels, durant laquelle de véritables flottilles, surtout américaines, pratiquèrent la chasse aux phoques et aux célacés dans le complexe très ramifié d'archipels auquel s'attache le nom du vieux marin légendaire Dirck Gerritsz. Si lon songe aux dangers de la navigation sous ces latitudes, par suite des glaces, des tempêtes subites, des brouillards et des grandes houles australes, un regard sur la carte suffira i montrer l'importance et la multiplicité des levés accomplis à diverses reprises au $\mathrm{S}$. du détroit de Drake par les baleiniers. A l'exception de Dumont d'Urville, Ross et Wilkes, dont les travaux dans ces parages furent d'ailleurs secondaires, tous les découvreurs ont été ici mûs surtout par le désir de rechercher la baleine franche ou de trouver de nouveaux repaires de phoques, depuis Smyth, Powell et Biscoe jusqu'i l'Allemand Dallmann'1(1873-187't) et aux croisières de l'“ Active " et du "Jason" (1892-1894). Il résulte de là que les levés se recommandent plutôt par le nombre que par la qualité : les chiservateurs étaient gênés dans leur curiosité par l'objet très pratique et nnercantile de leur mission ?

1. Mr Wichyasxil'etemanns Mitt., XLVII, 1901, p. 48) reproche à Mr DE Gerlache de ne s'être pas même demandé si lo détroit de Bismarck de Dallmann pouvait être identifié avec celui do la Belgica. L'orientation est en effet identique, et un examen de la carte prouve qu'il y a bien des chances que Dallmann ait retracé sous ce nom l'entrée Sud du détroit de la Belgica.

2. Le capitaine I Aarsev, du "Jason ", croisant en vuc des côtes de la Terro du Roi Oscar ('Terre de Graham Est), reconnait la possibilité de débarquer en plus d'un point de la côte: "Mais 
L'objet de la "Belgica "ílant purement scientitique, son ouvre topographique, pour restreinte qu'elle paraisse, semble par sa précision l'emporter sur celle des royageurs antérieurs. Les terres relevées sont presque

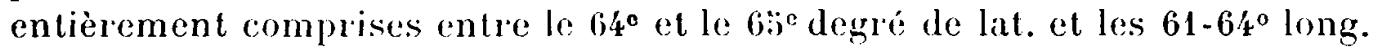
W. Gr. Elles ne correspondent quau pourtrour et aux îles de la baie Hughes (IIughes Inlet) of a un vaste delroit, inconnu auparavant, qui coupe et morcelle la terre jadis raguement appelée Terre de Palmer. L'aire explorée est limitée, a $\mathrm{S}$., au SW. el à l'W.par les découvertes de Biscoe el de Dallmann, au N. par les eaux bien connues des Shetlands, au NE. par le chenal d'Orléans et la Terrelouis-Philippe(Jumont d’urville), a l'E. par la grande trânée de terres du "Jetson "(189't). 11 n'y a pas de Terre de Palmer, mais un archipel inontagneux, assez fortement ramifić, et que coupe en deux le détroit de Gerlache ou de la Belgira, long de $8: 3$ milles marins, large en moyenne de 8 it 10 et oriente SW.-NE. Le Hughes Inlet n'est que l'entrée élargie du détroit, que parsèment des îles et des îlots, ot qu'échancrent de larges baies assez peu profondes : la plus longue, celle des Flandres, ne s'enfonce pas à plus de 12 milles. De loutes parts de grands glaciers débouchent dans ces échancrures. Les terres situés au $X$. du détroit garderont le nom d'archipel de Palmer; relles qui sont au S. forment la terre Danco, en sourenir de l'officiex, chargé des obserrations magnétiques, qui mourut pendant l'hivernage le :; juin 1898.

L'aspect de la carte i 1 : 462300 dressée par Mr Lecointe témoigne déjà de la minutie inusitée avec laquelle ce complexe d'ìles, de caps et de baies a ale releve. Scules les Shollands du Sud, sur une carte d'ensemble, apparaissent desormais aussi netlement connues. On n'en peut dire autant des Tores Louis-philippe, Joinville, Graham, des îles Biscoe et des terres du "Jason ". Liaure topographique y apparaît simplement ébauchée. L'expédition belge réussil d'ailleurs it opérer 20 débarquements dans le nouveau détroit; presque tous les accidents notables ou géographiquement intéressants ont été photographiés et l'on jugcra de la signification de ce détail, si l'on songe que la collection photographique de la "Belgica" est la première de cette importance que nous possédions des régions antarctiques. Le monde scientifique était dans une incroyable pénurie de photographies sur ce mystérieux domaine. Le live de Fricker: Antratis, a dû puiser exclusivement pour ses illustrations dans les collections de dessins de Dumont d'Urville, Ross, Hooker, du "Challenger ", etc. Les photographies de la "Belgica " sont donc une absolue nouveauté el, it bien des égards, une révélation.

phénomènes glaciaires. - C'est surtout au point de vue des phénomènes glaciaires que la photographie a été d'un précieux secours. On puise tout un enseignement en comparant la vue des caps et des monts les plus septentrionaux du détroit de la Belgica avec ceux du Sud, avoisinant les îles Biscoe ou la Terre de Graham. IJ'une façon générale, la ligne des neiges perpétuelles est extrèmement basse, et affleure le niveau de la mer dans les terres les plus meiridionales. En conséquence le sol tout entier y est couvert de névés ou de glace, seules les falaises verticales sont libres (île Cavelier

javais mission, dit-il, de cliasser les phoques et non de faire des découvertes géographiques it je dus refréner lo vif desir que javais de faire une excursion sur cette terre. "(Dr: (iERLachr, Note sur les expeditions... p. 409.) 
de Cuverville, Sierra Du Fief dans l'ile Wiencke). Ians le Nord au contraire, des respaces assez étendus sont libres, on relève de minces bandes côtières sans glace, où l'on peut aborder, et où quelques plantes infóricures poussent. Suivant l'orientation il y a des différences importantes. Alors que le Mi William (île Anvers) est entièrement grace, des caps comme le cap Renard et le cap Van Beneden, situés plus au S. pourtant, présentent de larges parois rocheuses qui se détachent en noir sur la blancheur des neiges. Au pied des falaises libres sadossent des glariers, formant le pied des monts, ou s'étalent sur des plages plus ou moins etendues de grands glaciers plats qui se brisent en tranche abrupte au bord de la mer. L'intérieur de la Terre Danco, vu du cap d'Lrsel, frésentait de même une immense etendue glacé i perte de vue, de surface horizontale, offrant les caracteres typiques d'une inlandsis. Ies exemples les plus etonnants de glaciation sont offerts par les petites îles. Relativement libres dans le Nord, elles sont entièrement envahies par les glaces dans les parages Sud, et alors on a res exemples si frappants de glaciers en forme de bonbonniere ou de camaper de tortue, au dos arrondi comme des conpoles très aplaties, chez qui la dimension est déterminée et réglée par l'écoulement lent de la glace, sous l’influence de la pesanteur, du centre vers la príriphérie. Les plus frappantes sont les îles Moureaux (baie des Flandres) et les îles Wauwermans (sortie Sud du détroit de la Belgica). Ce sont lí des transitions naturelles vers ces glaciers immenses de la Terre Alexandre, entrevue mystérieusement et de très loin par l'expédition, et "où less glaciers, séparés seulement par quelques arêtes à peine visibles, sont tous soudés vers le bas en un seul grand glacier, qui borle toute cette terre et forme un pied de glace qui plonge bien avant dans la mer ${ }^{1}$ ". On aurait donc dans la Terre Alexandre une sorte de pendant de la grande barrière de glace terrestre suivie par Russ et dernièrement par Burchgrevink, et dont l'origine a pendant si longtemps intrigue les geographes. A toutes les formes de transition dejat connues dans les régions boréales, on Srandinavie, au firoenland et dans la Terre Frangois-Joseph, si l'on ajoule les nouvelles decouvertes de: la "Belgica", le phénomène n'a plus rien que de très simple et de naturel.Les grands icebergs tabulaires s'expliquent aussi sans peine, par le détachement de blocs immenses appartenant à ces fronts glaciaires si reguliers. Plus on approchait du Nord, plus ces formes tabulaires se mélangeaient de formes déchiquetées et tourmenties, rappelant les icebergs arctiques. Ces différences ont leur explication aussi bien dans les changements d'équilibre dus à la fusion, que dans l'adjonction d'icebergs vomis par des glaciers de vallées et naturellement très déformés.

Découvertes océanographiques. - C'est dans ce domaine incontestablement que l'expédition belge apporte les résultats positifs les plus considirables. A part quelques sondages de Ross, nous ne possédions à peu priss aucune indication sur les mers antarctiques. la "Belgica" a d'abord opéré 8 sondages dans sa traversée du détroit de Drake. Elle s'est convaincue que: ce large bras de mer forme une cuvette ì fond légèrement relevé du N. vers le $\mathrm{S}$. et atteignant de 3700 i $4000 \mathrm{~m}$. au moins $\left(4040 \mathrm{~m}\right.$. par $36^{\circ} \mathrm{S}$. envi- 
ron et $63^{\circ} \mathrm{W}$. Gre, et 3690 par $61^{\circ}$ S. et le même méridien). Si donc l'on veut chercher dans l'archipel de Dirck Gerritsz une continuation des Andes de Patagonie qui disparaissent avec l'ile des États et le banc de Burdwood, il faudra etudier dans ce sens la Gérgie du Sud, le groupe des Sandwich et des South Orkney. Il y a la des sondages ì faire pour plus d'une expédition, mais il faut renoncer à trouver ce prolongement dans la liaison directe et méridienne des chaines américaines et des Andes Antarctiques (Antarctandes de $\mathbf{M}^{\cdot}$ Arctowski).

La séve principale des sondages a eté effectuée pendant les longs loisirs que créait la dérive dans le pack aux travailleurs de l'expédition. Loctanographie a sérieuscment profité de l'emprisonnement du navire. 30 sondages furent pratiques entre $69^{\circ} 06^{\prime}$ lat. S. et $71^{\circ} 33^{\prime}$ S., et $70^{\circ} 39^{\prime}$ et $102^{\circ} 1:^{\prime} \mathrm{W}$., dans la mer qui borde a l'W. la Tere Alexandre. Ces travaux ont démontré définitivement l'existence dans ces parages d'un plateau continental antarctique, preuve nouvelle et l'une des plus fortes qui aient encore été fournies de la rertitude d'un grand continent dans l'Extrême Sud. La plus grande partie de la dérive dans le pack se produisait sur le rebord de ce plateau, en sorte que les sondages les plus septentrionaux donnèrent 2600 et $2700 \mathrm{~m}$., les plus méridionaux, très nombreux, de 490 à $390 \mathrm{~m}$. In fait interessant et curieux, cest qu'ici c'est l'isobathe de: $; 00 \mathrm{~m}$. qui marque la bordure du plateau, at non pas, comme cest l'ordinaire, l'isobathe de $200 \mathrm{~m}$. Y a-t-il lit unc conséquence du raclage exercé par les grands icebergs, on ne sait. Ou bien le plateau continental antarctique est-il submergé, et dans ce cas, pourquoi recupe-t-il un niveau aussi bas ${ }^{1}$ ?

Le fond de la mer, au point de vue de la nature des sédiments, donna lieu ì de sérieuses études, fondées sur l'analyse mícanique, préconisée par $\mathrm{M}^{\mathrm{r}}$ Thoulet. $\mathrm{M}^{\mathrm{r}}$ Arctowski se rendit compte ainsi que la grande majorite de ces sédiments est de nature terrigine, et qu'ils ont éte apportés par les icebergs antarcliques. On releva au chalut des quantités énormes de cailloux erratiques, arondis, striés, noyis dans une vase plus ou moins épaisse, et qui ne peuvent provenir que des terres antaretiques situées au S. Ce sont lit des trouvailles auxquelles on s'altendait. Il n'en est pas de même de certaines vases calcaires qui, "si elles n'etaient pas mólangées ì des matières apportées, devaient etre classées parmi les vases à globigrérines ", sédiment pélagique de mer tempéré ou halde far excellence. Au contraire, on ne releva, maleré ce qu'on pouvait altendre, aucune vase à diatomées. In nouveau froblime de chimie sous-marine est ainsi posé, et une importante correction tevra être faite a lit carte des Deep Sea deposits de Nurray of Renard dans la collection du "Challenger".

$M^{r}$ Aretowski n'ayant pas encore fourni les mesures de saliniti, nous ne discuterons pas ici la superposition, cependant si interessante, des eaux en rouches chaudes wu froides.

Les effets physiologiques de la nuit polaire. - Il est d'ordinaire assez peu question, dans les récits de royages polaires, des allerations que la longue nuit entraine dans l'état sanitaireydes expéditions. Certaines d'entre clles

1. Par contre l'expedition allemande do la "Valdivia" a, comme on sait, dicouvert, au large de la Terre presumed dFnderby, une fosse de 5000 a $6000 \mathrm{~m}$. se reliant a locean Indien austral; et ici il faut substituer la notion diune mer profonie a l'hypothese d'un plateau sous-marin antaretique. Sans doute ce plateau se retrouve plus loin an Sud. 
semblent, il est vrai, être sorlies indemnes d'un ou mêne de plusieur's hivernages : les expéditions Niansen et Jackson en lémoignent. Encore ne peut-on plus guire douter que lés compagnons de Nansen aient été affectés dans leur moral ${ }^{1}$, et il se peut que les efrets physiologiques de l'obscurité aient contribué à cet énervement général qu'on a fini par nous avouer. Mais il est notoire qu'en bien des ras, la tin de l'hivernage a été marquée par de vraies catastrophes ou tout au moins par des cas de mort isolés. L'explication ordinaire, dont on se contente, est l'explosion du scorbul, causcé par un régime alimentaire mal compris. Le scorbut est en effet l'une des causes principales des filats morbides graves qu'on constate en pareille occurrence, mais ce n'est pas la seule.

Par une bonne fortune assez rare dans la littírature des voyages aux regions glaciales, la relation la plus copieuse que nous possédions de l'expédition belge est due au médecin de la "Belgiea ". Yous devons au Dr Conk une: sćrie très intéressante de noles qui prouvent qu'à l'instar des plantes élevées en cave, les hommes privés pendant plusieurs mois de la bienfaisante lumière du soleil subissent une série de troubles physiologiques caractérisés. L'expédition de Gerlache resta 66 jours, du 16 mai 1898 au 22 juillet, sans voir le globe du soleil. Jusqu'au $1^{\text {er }}$ mai, c'est-it-dire jusqu'aux premieres approches de la nuit continue, la santé générale avait été excellente. C'est alors que les premiers indices de dépression physique, morale et aussi mentale,commencent à siacuser. Les habitants de la "Belgica" perdent l'appétit, marquent de l'impatience pour la société de leurs compagnons et une mauraise humeur générale. Les troubles physiques sont des plus curieux. "Physiquement nous perdons de plus en plus nos forces, bien que notre poids reste ì peu près le mème; chez quelques-uns mème il augmente. Chez tous, il se produit des bouffissures autour des yenx ot aux rhevilles; les muscles, fermes nagnire, sont mous, toutefois sans perdre de leur volume. Nous sommes palles; notre peau est plus huileuse que de coutume. Les cheveux poussent avec rapidité, el la peau qui entoure les ongles a tendance à les recouvrir, comme pour les protéger du froid. L'action du cœur perd de sa force et est nettement irrégulière. La moindre excitation affecte cet organe d'une manière alarmante. Une promenade un peu précipitée autour du bateau fait monter le pouls ì 110 pulsations; si l'on continue un quart d'heure, il cesse de battre, et l'on éprouve quelque peine à respirer. Les observateurs qui vont aux observatoires, situés ì quelque $100 \mathrm{~m}$., reviennent de ce court voyage presque hors d'haleine. Le pouls est d'ailleurs variable d'un jour à l'autre. Tantòt il est plein, régulier, vigoureux, tantôt mou, faible et intermittent. Hier on relevait 4.3 pulsations, aujourd'hui c'est 98 . L'homme

1. Cela ressort de toute evilonce des renscignements de Nordaur et Jaconsks. IJe Dr Cook reproche avec vivacité a Nansen davoir passe sous silence dans son livre tout co qui concerne lietat sanitaire de son équipagre: « Il se borne, dit-il, à quelques remarques fanfaronnes (boastful) sur les effets physiques de la nuit arctique. et il conclut, pour se faire valoir lui-méme, qu'ils nont eprouve aucun des malaises habituels; mais depuis qu'on a su quin de ses meillours hommes est revenu avec des troubles mentaux - ce que Nansen nous a laissé ignorer nous pouvors inferer que d'autres taits analogues ont pu aussi lui sortir de la mémoire. Il n'est pas possible a une expedition de douze hommes do vivre trois ans dans les régions arctiques sans quelques indispositions physiques "(Cook, p. 321). Il est juste de dire, pour la justification de Nansen, que colui-ci annonco pour la prétaco do son grand ouvrage (The Norwegian North Polar Ixpedition, I, p. Iv) des Physiological investigations made on board the Fram, du Dr Blesssivg, et une monographie du scorbut arctique, par Sorrus Torur. 
ne se plaint de rien et vaque à son labeur habituel. Il semble que le soleil fournisse quelque chose d'indétinissable qui rìgle et tempère le caur. En son absence, il fonctionne comme une machine sans regulateur ". In triste crénement vint demontrer la vérite de cette observation; le lieutenant Daneo, qui était alteint d'une hypertrophie du corur, dont il ne souffrait point dans les anditions numales de la vie, stleromba le . juin, apres avoir décline rapidement el sans remele, à mesture que s'epalssissait la nuit.

Tous aes symptomes fumbentence agglaves par labus des conserves,

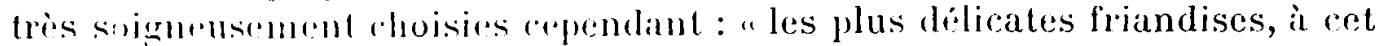
cigard, des marches de Belgique, de France ou de Norvege, mixtures de laboratoire, hat:his aux noms alléhants, boulettes de viande et de poisson, etr. ". Or "apres sept ou huit mois, il y a quelque chose dans l'organisme humain qui se refuse à absorber relle sorte de mets ". La chair des manchots, si distestable qu'elle soit, sauva la vie à plusieurs membres de l'equipage, re qui s'accorde arec l'expérience que Nansen et Johansen avaient déja faite dans la Terre de François-Joseph, en se nourrissant lout un hiver de chair d'ours el de phoque. $A$ près le retour du soleil, en aoùt et en septembre, l'expedition antarctique abandonna presque complètement les conserves el adopta pour unique régime la viande fraîche de phoque on de manchot. Ce fut lì, avec l'emploi aussi prolongé que possible de glauds feux de charbon, donnant ì la fois abondance de chaleur et de lumière, liss princinaux remèdes par lesquels on combattit les redoutables effets de $\cdots / 10$ "anémie polaire" que le $\mathrm{D}^{\mathrm{r}}$ Cook décrit à plusieurs reprises avec tanl de netteté. Jamais, assure-t-il, il n'en a observé des manifestations aussi inquietantes, soit lors de son expérience personnelle avec Peary, soit dans les relations sérieuses de témoins compétents. Ces lroubles, compliqués d'allerations mentales, d'insomnies, se prolongèrent jusque fort avant dans le printemps. Plusieurs hommes, au mois d'aoùt encore, témoignèrent de dérangements cérébraux, et l'un d'eux donna des signes de folie qui ne se dissipèrent que peu à peu.

N'y aurait-il pas quelque fruit à comparer l'anŕmie "polaire " à l'anémie "tropicale"?

Il y aurait lieu d'étudier de la même manière bien d'autres points élucidés par a voyage, et nolamment les constatations si curieuses de IIr Racovitza sur la biologie, les résultats météorologiques capitaux de ce premier hivernage dans le monde austral, la dérive et le régime des glaces de mer, les constatations géologiques. Mais il nous semble qu'il est préfírable de les réserver pour un travail d'ensemble. On voulait simplement signaler ici la portée si grande dans la science géographique de ce voyag" qui pourtant, au regard des résultats obtenus, a coûté si peu.

Malitice Zimuerman. 